\title{
El capital social de los hijos de inmigrantes procedentes de Rumanía, Ecuador y Marruecos en España
}

\author{
Francisco Javier de Lorenzo Gilsanz \\ florenzo.ssgg@caritas.es \\ Fundación FOESSA
}

\begin{abstract}
Resumen: El término capital social es uno de los más utilizados en la literatura especializada de las últimas décadas. De hecho, resulta un concepto claramente evocador de diferentes contenidos pero difícilmente medible, en función de la definición que se haga de él. A partir de un trabajo de desagregación en componentes y de una propuesta metodológica concreta, hemos tratado de elaborar un índice sintético para medir el capital social y otro para los bienes relacionales. El contenido fundamental de este artículo recoge dicha propuesta y su aplicación sobre jóvenes (entre 18 y 29 años) que, teniendo o no la nacionalidad española, cuenten con un pasado migratorio familiar reciente (es decir, hijos de inmigrantes) y que residan desde hace un mínimo de 15 años en nuestro país. Además, se ha establecido una comparativa entre quienes constituyen nuestro campo de estudio y otros dos grupos distintos con los que comparten características importantes pero se diferencian en aspectos significativos: adultos inmigrantes residentes en España con los que comparten procedencia cultural y jóvenes españoles de entre 18 y 29 años sin pasado migratorio familiar reciente con los que comparten edad y, en muchos casos, nacionalidad.
\end{abstract}

Palabras clave: Capital social, bienes relacionales, integración, exclusión, migrantes.

Abstract: The term social capital is one of the most used in the specialized literature of recent decades. In fact, it is a concept clearly reminiscent of different contents, but difficult to measure, depending on the definition used. From a work of disaggregation into components and a concrete methodological proposal, we have tried to elaborate a synthetic index to measure the social capital and another one for the relational goods. The fundamental content of this article includes this proposal and its application to young people (between 18 and 29 years old) who, with or without Spanish nationality, have a recent past of family migration and a minimum of 15 years in our country. In addition, a comparison has been estab- 
lished between those who make up our field of study and two other groups with whom they share important characteristics but differ in significant aspects: immigrant adults living in Spain with whom they share cultural background and young Spaniards between 18 and 29 years old without recent family migration past with whom they share age and, in many cases, nationality.

Keywords: Social capital, relational goods, integration, exclusion, migrants. 


\section{CAPITAL SOCIAL: UN CONCEPTO, DISTINTAS PERSPECTIVAS ${ }^{1}$}

El término capital social es uno de los más utilizados en la literatura especializada de las últimas décadas. En 2003, Putnam señalaba que «Una búsqueda en la bibliografía sociológica internacional dio como resultado 20 artículos dedicados al capital social antes de 1981, 109 entre 1991 y 1995, y 1003 entre 1996 y marzo de 1999» ${ }^{2}$.

Resulta complicado hacer una estimación, ni siquiera aproximada, de cuántas referencias podríamos encontrar a día de hoy, pero es seguro que superarían con creces las cifras del pasado. Así, el uso de este término no solo es habitual, sino que ocupa un lugar destacado en las ciencias sociales.

Por lo tanto, podríamos afirmar que existe una gran producción bibliográfica que se adentra en el concepto hasta situarlo en el panorama intelectual internacional de manera consolidada. Los esfuerzos dedicados por sociólogos de prestigio como Pierre Bourdieu, James S. Coleman, Robert D. Putnam o Alejandro Portes así lo acreditan. Pero, en las teorías fundamentales sobre capital social, ¿se parte de un mismo concepto o en realidad se está hablando de fenómenos diferentes?; dicho de otra forma, ¿existe relación entre la cultura democrática de un país y las oportunidades potenciales a las que accede una persona a través de su círculo de relaciones?, ¿entre el papel que desempeña la sociedad civil y los privilegios que residen en la pertenencia a un grupo determinado?

De forma general, el capital es entendido como el factor que, en colaboración con otros (principalmente el trabajo), se destina a la producción de bienes. Es decir, bienes producidos que, a su vez, sirven para crear nuevos bienes. Si entendemos las relaciones sociales como bienes capaces de concebir otros diferentes, podríamos asumir que, efectivamente, lo social tiene un carácter productivo capaz de generar capital. Ahora bien, esto supondría asumir un riesgo importante: reducir todo lo relacional a elementos de producción. Lo cual conlleva una mirada sesgada y claramente incompleta, pues obviaría aquellos elementos que se generan en las dinámicas relacionales y no son convertibles en unidades económicas. Elementos como el afecto, la construcción de identidad grupal, el sentido vital o la pertenencia son bienes generados y compartidos en el seno de las relaciones sociales, pero convertirlos en capital supondría vaciarlos de significado, pues entrañaría limitar su capacidad de satisfacer otro tipo de necesidades funda-

${ }^{1}$ Este apartado es una síntesis elaborada a partir del artículo LORENZO, F. (2017): «¿Capital social o bienes relacionales? Elaboración de una propuesta conceptual del capital social» en Documentación Social n. ${ }^{\circ}$ 185. Cáritas Editores. Madrid.

2 PUTNAM, R.D. (2003): El declive del capital social. Un estudio internacional sobre sociedades y el sentido comunitario. Barcelona: Galaxia Gutemberg. 
mentales para todo ser humano, independientemente del momento y el lugar en el que se encuentre.

Únicamente algunos de esos bienes sustentados en las relaciones sociales constituyen capital en sentido estricto, pues resultan fundamentales a la hora de explicar la activación de procesos de movilidad ascendente ${ }^{3}$ en los que juegan un papel claramente reconocible.

Estos bienes cuentan con un elevado potencial en términos de lucha contra la exclusión o en la satisfacción de necesidades humanas, pero no son equivalentes a los que podemos considerar capital. Esta decisión se basa en la necesidad de un proceso previo de transformación de esos bienes. Mientras que los componentes en los que hemos desagregado el capital social están disponibles de forma inmediata para su uso (aprovechamiento), los denominados bienes relacionales requieren de reelaboración previa y de un proceso de «fermentación» que nos lleva a considerarlos como bienes intermedios ${ }^{4}$.

De forma esquemática, podemos establecer la siguiente diferenciación:

\section{TABLA 1}

Capital social y bienes relacionales

\section{Capital social}

Bienes o servicios materiales (ayuda material).

Información.

Influencia y acceso a otras relaciones.

\section{Bienes relacionales}

Bienes socioemocionales (afecto y seguridad).

Marcos de referencia y sentido vital:

— Visión del mundo/valores.

— Visión de uno mismo/identidad.

Fuente: Elaboración propia

Por todo lo visto hasta este momento, podemos afirmar entonces que el capital social es el agregado de bienes materiales, de información y de influencia y red de contactos que los miembros de un grupo ponen a disposición del resto de integrantes. Para que dicho capital sea accesible se deben dar una serie de condiciones dentro del grupo. Solo

${ }^{3}$ Por movilidad social entendemos aquellos procesos de traslación de los individuos entre diferentes posiciones socioeconómicas dentro de la estratificación de una sociedad.

${ }^{4}$ A modo de ejemplo, podemos afirmar que un nivel adecuado de autoestima (que podríamos considerar un bien relacional producto de unas relaciones estimulantes y que reconozcan las capacidades de la persona) nos habilita para ser más competitivos en el mercado laboral; ahora bien, el aprovechamiento de este recurso requiere de otros complementarios y de cierta «manufactura» antes de transformarse en el acceso a un puesto de trabajo (y por lo tanto en un bien capitalizable de forma inmediata). 
entonces se producirá circulación de dicho capital, de manera que se puedan establecer dinámicas de movilidad social ${ }^{5}$.

Tras una primera fase de análisis y reelaboración conceptual, resulta imprescindible llevar a cabo su aplicación sobre una realidad concreta, ya que a través de su aplicabilidad podrá cumplir una función que vaya más allá de su capacidad metafórica o de su virtud polisémica. En nuestro caso, lo haremos sobre un grupo: los jóvenes con pasado migratorio familiar reciente, los cuales configuran una realidad suficientemente homogénea como para que el concepto pueda ser desarrollado de manera rigurosa; pero, a su vez, hemos buscado diferencias internas suficientes (pues hemos seleccionado a aquellos con procedencia familiar de Ecuador, Rumanía y Marruecos) que nos permitan la depuración del mismo. Además, hemos llevado a cabo una comparativa con otros dos grupos: sus progenitores y los jóvenes nacidos en familias españolas sin pasado migratorio reciente (españoles hijos y nietos de españoles).

La pregunta inicial que guía este trabajo nos plantea si «la cantidad de capital social» a la que tienen acceso estos jóvenes guarda relación con su nacionalidad o viene determinada por la cantidad de recursos que tienen principalmente sus progenitores, amigos, vecinos... Y en un segundo momento, si aquellas personas que tienen una relación de calidad con personas en posiciones socioeconómicas claramente superiores tienen un acceso potencial a niveles de capital social más elevados independientemente de otras variables.

\section{LA MEDICIÓN DEL CAPITAL SOCIAL ${ }^{6}$}

El proceso de medición del capital social se hará partiendo del análisis desagregado de sus componentes ${ }^{7}$. Este proceso se llevará a cabo teniendo en cuenta quiénes integran la red social de una persona, qué posición social ocupan y cómo son estas relaciones. Son estos tres factores los que nos van a permitir la construcción de un índice sintético básico.

${ }^{5}$ En la generación de capital social juegan un importante papel las características del contexto en el que se producen las relaciones. Así, nos podemos encontrar contextos catalizadores del capital social, contextos neutros y contextos inhibidores del capital social en función de si son generadores de movilidad descendente, de estancamiento o de movilidad ascendente. Para ampliar información ver LORENZO (2017).

${ }^{6}$ En LORENZO (2015) se recoge un desarrollo ampliado de lo expuesto en el presente epígrafe.

7 Dado que también nos interesa el acceso a otros elementos que intervienen directamente en la situación y en los procesos de exclusión/integración, haremos un análisis similar relativo a los bienes relacionales ya mencionados (apoyo afectivo, valores e identidad). 


\section{Primer factor: los bienes asociados a las relaciones}

El primer factor que vamos a analizar hace referencia a los bienes y a los recursos que, de forma habitual, se encuentran asociados a determinadas relaciones.

Wellman (1991) realiza una propuesta basada en diversos estudios, a través de la cual establece una correspondencia (más o menos reiterada) entre algunas relaciones sociales y determinado tipo de recursos. A partir de sus aportaciones podemos elaborar la siguiente representación:

\section{TABLA 2}

Relaciones sociales y tipos de bienes provistos por los distintos grupos de relaciones

\begin{tabular}{|c|c|c|c|c|c|c|}
\hline & \multicolumn{3}{|c|}{ CAPITAL SOCIAL } & \multicolumn{3}{|c|}{ BIENES RELACIONALES } \\
\hline & $\begin{array}{c}\text { Apoyo } \\
\text { material }\end{array}$ & Información & Influencia & Afecto & Valores & Identidad \\
\hline $\begin{array}{l}\text { Familia } \\
\text { directa }\end{array}$ & Habitual & Habitual & Habitual & Habitual & Habitual & Habitual \\
\hline $\begin{array}{l}\text { Familia } \\
\text { extensa }\end{array}$ & Ocasional & Ocasional & Ocasional & Habitual & Ocasional & Habitual \\
\hline Amigos/as & Ocasional & Habitual & Habitual & Habitual & Habitual & Habitual \\
\hline Vecinos/as & Ocasional & Ocasional & Ocasional & Inusual & Habitual & Habitual \\
\hline Otros & Inusual & Habitual & Ocasional & Inusual & Ocasional & Inusual \\
\hline
\end{tabular}

Fuente: Elaboración propia

Así, a cada intersección producida al cruzar grupos (familia directa, extensa, amistades...) con tipo de bienes/recursos (ayuda material, información, influencia...) le corresponde una frecuencia establecida: habitual, ocasional o inusual. En nuestra propuesta inicial, hemos asignado los siguientes valores a estas tres categorías:

\section{ESQUEMA 1}

Escala de valores asignados en función de la frecuencia relacional

\begin{tabular}{lccr} 
Inusual & Ocasional & Habitual \\
\hline 0,3 & 0,6 & 1
\end{tabular}

Fuente: Elaboración propia.

Hemos decidido realizar una escala de valores sencillos (de 0,3 a 1), y hemos considerado que entre categorías consecutivas se produce un incremento considerable de la 
frecuencia relacional; por ello, hemos asignado a cada categoría casi el doble del valor que tiene la inmediatamente anterior. Así, la matriz resultante sería la siguiente:

TABLA 1

Relaciones sociales y valores asignados al capital social y los bienes relacionales provistos

\begin{tabular}{lcccccc}
\hline & \multicolumn{3}{c}{ CAPITAL SOCIAL } & \multicolumn{3}{c}{ BIENES RELACIONALES } \\
\cline { 2 - 7 } & $\begin{array}{c}\text { Apoyo } \\
\text { material }\end{array}$ & Información & Influencia & Afecto & Valores & Identidad \\
\hline Familia directa & 1 & 1 & 1 & 1 & 1 & 1 \\
Familia extensa & 0,6 & 0,6 & 0,6 & 1 & 0,6 & 1 \\
Amigos/as & 0,6 & 1 & 1 & 1 & 1 & 1 \\
Vecinos/as & 0,6 & 0,6 & 0,6 & 0,3 & 1 & 1 \\
Otros & 0,3 & 1 & 0,6 & 0,3 & 0,6 & 0,3 \\
\hline
\end{tabular}

Fuente: Elaboración propia

Nos hemos decantado por una escala cuya puntuación inferior fuera 0,3 (en lugar de 0) dado que, para la desagregación de casos en diferentes categorías relativas a intervalos de puntuaciones, los valores nulos pueden generar determinados problemas a la hora de realizar operaciones matemáticas. Además, no hemos querido identificar la categoría menor (inusual) con otra inexistente (la categoría nunca) con la que se correspondería mejor el valor 0 , ya que cualquier relación potencialmente puede conllevar excepciones.

Ahora bien, el hecho de utilizar el valor 0,3 como mínimo nos obliga a establecer un umbral de corte superior al 0 , el cual se desprenderá del análisis de datos llevado a cabo $^{8}$.

\section{Segundo factor: calidad y frecuencia en las relaciones}

El segundo factor se elabora a partir del siguiente cuadro:

${ }^{8}$ A pesar de lo dicho, resulta obvia la imposibilidad de definir una linealidad absoluta en la correlación planteada por Wellman. Por este motivo, procedería confeccionar un modelo numérico en el que los valores asignados de forma estándar resultaran de testar un número de casos elevado. Ante la inexistencia de dichos datos, y como modelo experimental, nos decantamos por utilizar valores fijos, conscientes de estar asumiendo una primera limitación a la hora de representar, lo más fielmente posible, el capital social de una persona. 
CUADRO 3

Relaciones sociales y movilidad social

\begin{tabular}{llll}
\hline & \multicolumn{1}{c}{$\begin{array}{c}\text { Movilidad } \\
\text { descendente }\end{array}$} & Estancamiento & \multicolumn{1}{c}{$\begin{array}{c}\text { Movilidad } \\
\text { ascendente }\end{array}$} \\
\hline Fortaleza relacional & $\begin{array}{l}\text { Desafiliación/ } \\
\text { filiación tóxica }\end{array}$ & Débiles & Fuertes/débiles \\
$\begin{array}{l}\text { Diversidad relacional } \\
\text { Dinámica relacional }\end{array}$ & $\begin{array}{l}\text { Inexistentes-nocivas } \\
\text { Inhibidoras }\end{array}$ & $\begin{array}{l}\text { Muy homogéneas } \\
\text { Poco activas }\end{array}$ & $\begin{array}{l}\text { Heterogéneas } \\
\text { Muy activas }\end{array}$ \\
\hline
\end{tabular}

Fuente: Elaboración propia

Si bien, de forma habitual, determinadas relaciones encierran la potencialidad de ser proveedoras de determinados recursos, su calidad hace que estén más o menos activadas. Expresado a través de un ejemplo, es fácil comprender que, a pesar de que las amistades pueden ofrecer de manera ocasional algún tipo de información relevante (capitalizable), si la relación es infrecuente o excesivamente débil, la probabilidad de que esto ocurra es claramente menor.

La escala empleada para este factor sigue una lógica similar a la del factor anterior, pero con dos matices: consideramos que la calidad y frecuencia de las relaciones no incrementan los valores del factor previo, sino que, en el mejor de los casos, unas relaciones muy fuertes y muy activas permitirán sacar el máximo partido existente. Por lo tanto, a esta situación le concedemos el valor 1. Entendemos que ni siquiera el mejor supuesto genera nuevos recursos (si así fuera, le habríamos asignado un valor superior a 1), sino que habilita para el máximo acceso a los que potencialmente hay.

El segundo matiz tiene que ver con que, en esta ocasión, sí podremos emplear el valor 0 , pero únicamente para aquellos casos en los que no exista una relación determinada (por lo tanto no encierra ni siquiera una potencialidad). A modo de ejemplo, resulta sencillo entender que aquel que no tiene familia extensa no puede esperar de ella recurso alguno, ni en el presente ni en el futuro. En el caso de existir relación, pero ser débil o poco activa, recibirá la puntuación 0,1 .

ESQUEMA 2

Escala de valores asignados en función de la fortaleza relacional

\begin{tabular}{lcccc}
$\begin{array}{l}\text { Relaciones débiles/ } \\
\text { poco activas }\end{array}$ & $\begin{array}{c}\text { Relaciones } \\
\text { fuertes/activas }\end{array}$ & $\begin{array}{r}\text { Relaciones muy } \\
\text { fuertes/muy activas }\end{array}$ \\
\hline 0,1 & $\mathrm{l}$ & 0,5 & $\mathrm{l}$ & 1
\end{tabular}

Fuente: Elaboración propia. 
Una escala de valores mejorada sería continua, pudiendo asignarse valores intermedios entre el 0,1 y el 1. En nuestro caso, nos hemos decantado por utilizar únicamente los tres valores recogidos en el esquema, ya que la asignación de valores intermedios se debería llevar a cabo con criterios claros y objetivos; es decir, estaríamos obligados a crear una serie de categorías bien descritas que permitieran distinguir claramente las características de dicha relación ${ }^{9}$.

\section{Tercer factor: posición socioeconómica}

El último de los factores considerados en este modelo agregado hace referencia a la posición socioeconómica de un individuo en comparación con la de cada uno de sus grupos de relación. La premisa de partida que encontramos detrás de la construcción de esta escala es que los recursos a los que se tiene acceso mediante las redes que se encuentran en posiciones socioeconómicas similares aportan escasos márgenes de movilidad social. $\mathrm{Su}$ efecto se reduce a lo que podríamos denominar como apoyo meramente instrumental.

Ahora bien, entrar a formar parte de redes que cuentan con una posición superior incrementa considerablemente el potencial de acceso a recursos capitalizables. Trasladado a ejemplos concretos, esto supondría que una persona en desempleo que se relaciona con amistades en una situación similar difícilmente va a recibir de estas información privilegiada que le permitan encontrar un puesto de trabajo.

Por su parte, entrar en «un circuito relacional» en el que existen recursos mejores que aquellos con los que cuenta una persona le habilita a acceder a ellos (dependiendo, eso sí, de los dos factores mencionados anteriormente) y a posibles procesos de mejora socioeconómica.

De ahí que la escala, en esta ocasión, se construya de forma similar a las anteriores (obviando el valor 0 por los motivos explicados para el factor primero), pero alcanzando el valor 2 como el superior de la escala.

\section{ESQUEMA 3}

Escala de valores asignados en función de la posición socioeconómica

\begin{tabular}{lcr}
$\begin{array}{l}\text { Posición social } \\
\text { inferior }\end{array}$ & $\begin{array}{c}\text { Posición social } \\
\text { semejante }\end{array}$ & $\begin{array}{r}\text { Posición social } \\
\text { superior }\end{array}$ \\
\hline 0,5 & 1 &
\end{tabular}

Fuente: Elaboración propia

\footnotetext{
${ }^{9}$ Para llevar a cabo este proceso, una vez más, necesitaríamos mayor cantidad de registros con información.
} 
Este factor supone, en realidad, la cantidad de recursos que se ponen a disposición de una persona gracias a sus relaciones. Dicha cantidad se verá afectada por su calidad y frecuencia y por el grupo como potencial proveedor de estos recursos.

\section{Modelo de combinación de factores}

Una vez descritos los tres factores que integran el índice sintético de capital social, es necesario detallar cómo interrelacionan para ofrecer un valor concreto. Se trata, por tanto, de establecer un modelo de combinación que nos permita determinar qué obtiene cada persona (y en qué medida) de cada uno de sus grupos de relación, lo cual, como hemos dicho, nos permitirá medir la cantidad de capital social (agregado de sus tres componentes) y tratar de replicarlo para obtener una puntuación comparativa de los bienes relacionales que posee.

El objetivo es obtener una matriz similar a la Tabla 2 en la que contemos con una puntuación para cada una de las intersecciones (celdas) entre grupos de relación y componentes desagregados de capital social y bienes relacionales. Es decir:

\section{TABLA 2}

Relaciones sociales y tipos de bienes provistos. Valores obtenidos

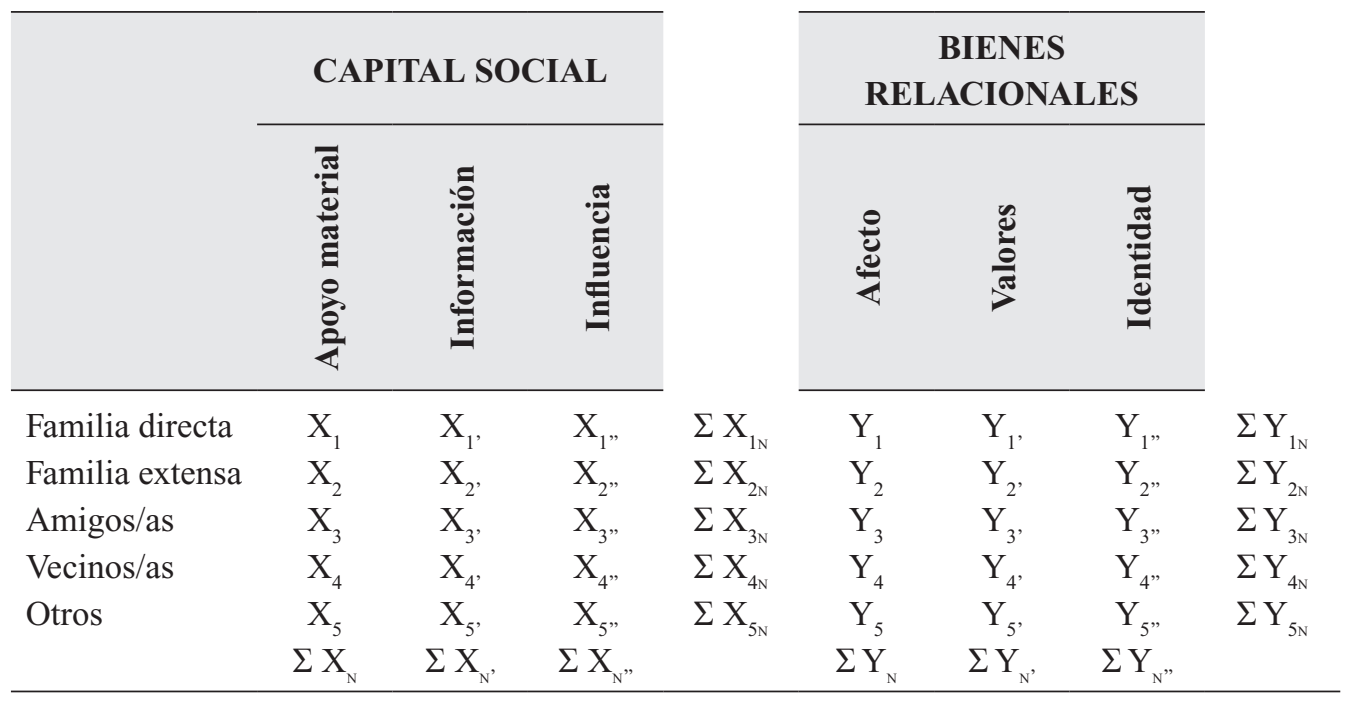

Fuente: Elaboración propia

Esto nos permitirá realizar dos tipos de mediciones a través de sumatorio: por componente y por grupo de relaciones. Es decir, podremos conocer cuánto «apoyo material» 
tiene disponible una persona a través de todos sus grupos de relación, y también cuánto capital social está potencialmente disponible a través de sus amistades. Valores similares se pueden obtener para cada grupo, para el capital social, los bienes relacionales y para cada uno de los componentes desagregados que los integran.

Para calcular cada una de las puntuaciones de las distintas intersecciones señaladas, multiplicaremos el valor de los tres factores correspondientes.

A modo de ejemplo, obtendremos el resultante del apoyo material al que potencialmente tiene acceso una persona a través de su grupo de vecinos multiplicando el valor obtenido para su factor primero (en este caso el valor genérico sería 0,6 ) por el del factor segundo ( 0,5 en el caso de que las relaciones establecidas sean fuertes y activas) y por el del factor tercero ( 2 en el caso de que se encuentren en una posición socioeconómica claramente superior a la del individuo en cuestión).

La resultante expresará cómo se combinan estos tres factores, siendo la posición socioeconómica la cantidad de recurso disponible, el cual estará más o menos (potencialmente) accesible en función de qué grupo sea el que cuenta con dicho recurso y cómo sea la relación que tenemos con él ${ }^{10}$. Una vez realizados los sumatorios correspondientes, podremos realizar comparativas de diversos tipos ${ }^{11}$.

\section{ANÁLISIS COMPARATIVO DEL CAPITAL SOCIAL Y LOS BIENES RELACIONALES DE LOS HIJOS DE INMIGRANTES PROCEDENTES DE RUMANÍA, ECUADOR Y MARRUECOS EN ESPAÑA}

\section{Campo de estudio}

El campo de estudio que da lugar a este artículo está constituido por jóvenes (entre 18 y 29 años) que, teniendo o no la nacionalidad española, cuenten con un pasado migratorio familiar reciente (es decir, hijos de inmigrantes) y que residan desde hace un mínimo de quince años en nuestro país ${ }^{12}$. Hemos considerado que este plazo establece la frontera para la construcción de un proyecto de enraizamiento; es decir, quienes permanecen durante al menos este tiempo en un país residen durante un periodo suficiente como para que se puedan llevar a cabo procesos de acceso y acumulación de capital social a través de la construcción de un tejido relacional más o menos estable.

\footnotetext{
${ }^{10}$ Como ya hemos venido explicando, el valor 0 solo se asignará para aquellos grupos no existentes.

${ }^{11}$ En nuestro caso concreto, en el capítulo siguiente las haremos entre cada uno de los individuos entrevistados, en función de su grupo de edad o de su procedencia migratoria, por componentes y por grupos de relación.

12 De aquí en adelante nos referiremos a ellos como Grupo 1.
} 
Además, dadas las edades a las que nos referimos, nos estaremos encontrando con jóvenes cuya identidad se verá probablemente orientada por dos «vectores» que ejercen fuerzas considerables (en sentidos si no opuestos, al menos sí relativamente contradictorios): llevar un elevado porcentaje de su vida en España, por un lado, y su origen familiar extranjero, por otro.

Para poder identificar las variables dependientes e independientes, se ha establecido una comparativa entre quienes constituyen nuestro campo de estudio (Grupo 1) y otros dos grupos distintos con los que comparten características importantes pero se diferencian en aspectos significativos. En primer lugar, con el grupo de adultos inmigrantes residentes en España (Grupo 2) comparten procedencia cultural o, al menos, cierta herencia. Por su parte, con los jóvenes españoles de entre 18 y 29 años sin pasado migratorio familiar reciente (Grupo 3) comparten edad y, en muchos casos, nacionalidad.

Dadas las posibles diferencias que pueden derivarse de la nacionalidad (o del país de origen), nos hemos decantado por trabajar sobre tres concretas en función de la procedencia: Ecuador, Rumanía y Marruecos ${ }^{13}$. Por una parte, los tres países mencionados son los que más personas aportan a las cifras totales de inmigrantes en España. Pero, además, nos interesan por contar (previsiblemente) con diferente capital social en función de sus idiomas de origen, de sus distintos tratamientos jurídicos, del desigual grado de aceptación cultural con el que cuentan en nuestro país y de las diferencias en el acceso al mercado laboral. Las desemejanzas existentes ofrecen gran variedad de características que los hacen suficientemente representativos del total de inmigrantes ${ }^{14}$.

El cruce de grupos y subgrupos permite que entren en juego aspectos relativos a la apariencia, la edad, la identidad y la nacionalidad que configura nuestro objeto de estudio (Grupo 1) como un grupo vinculado íntimamente a los otros dos, pero sin llegar a producirse una identificación plena; más bien al contrario, produciéndose una diferenciación que les obliga a encontrar su propia forma de identificarse o, en términos de identidad, a «transitar por tierra de nadie».

Por último, se ha tenido en cuenta otra variable a la hora de establecer distintos análisis comparativos: el nivel socioeconómico. Para ello, se ha utilizado uno de los tres componentes del indicador AROPE ${ }^{15}$ utilizado por la UE. En concreto, se ha empleado

${ }^{13}$ A los que denominaremos subgrupos.

${ }^{14}$ Obviamente, no nos referimos a representatividad estadística, sino a su valor característico en cuanto a tipología de perfiles.

${ }^{15}$ En sus siglas en inglés: At Risk of Poverty and/or Exclusion. http://ec.europa.eu/eurostat/statistics-explained/index.php/Glossary:At_risk_of_poverty_or_social_exclusion_(AROPE), acceso en 6 de agosto de 2015 . 
el componente de baja intensidad laboral que engloba a aquellos hogares que trabajaron menos del $20 \%$ de su tiempo potencial de trabajo durante el año pasado.

Además de las características señaladas anteriormente, resulta apropiado tener en cuenta la posición socioeconómica que se ocupa, puesto que esta (previsiblemente) facilita un acceso a distinto (tipo y cantidad de) capital social. Por ello, hemos establecido dos umbrales específicos. Teniendo en cuenta todas las variables mencionadas, se realizaron 33 entrevistas distribuidas de la siguiente forma:

\section{CUADRO 4}

Entrevistas realizadas

\begin{tabular}{|c|c|c|c|}
\hline Grupo & Subgrupo & $\begin{array}{c}\text { Baja intensidad } \\
\text { laboral }\end{array}$ & $\begin{array}{c}\text { Media-alta } \\
\text { intensidad laboral }\end{array}$ \\
\hline \multirow{7}{*}{$\begin{array}{l}\text { Inmigrante o español con } \\
\text { pasado migratorio familiar } \\
\text { reciente. }\end{array}$} & 1.1 & E07 (Mujer) & E27 (Mujer) \\
\hline & Ecuador & E22 (Hombre) & E30 (Hombre) \\
\hline & 1.2 & E17 (Hombre) & E04 (Mujer) \\
\hline & Marruecos & E20 (Mujer) & E33 (Hombre) \\
\hline & 1.3 & E02 (Mujer) & E16 (Hombre) \\
\hline & Rumanía & E09 (Mujer) & E29 (Mujer) \\
\hline & & E23(Hombre) & \\
\hline \multirow{8}{*}{$\begin{array}{c}\text { Grupo } 2 \\
\text { Adulto. } \\
\text { Inmigrante. }\end{array}$} & 2.1 & E15 (Hombre) & E11 (Mujer) \\
\hline & Ecuador & E25 (Mujer) & E12 (Mujer) \\
\hline & 2.2 & E13 (Mujer) & E14 (Hombre) \\
\hline & Marruecos & E18 (Mujer) & E21 (Hombre) \\
\hline & & E19 (Hombre) & \\
\hline & 2.3 & E01 (Mujer) & E05 (Hombre) \\
\hline & Rumanía & E08 (Mujer) & E10 (Hombre) \\
\hline & & E24 (Mujer) & \\
\hline Grupo 3 & - & E26 (Hombre) & E03 (Hombre) \\
\hline De 18 a 29 años. & & E28 (Mujer) & E06 (Mujer) \\
\hline $\begin{array}{c}\text { Español sin pasado migratorio } \\
\text { familiar reciente. }\end{array}$ & & E31 (Mujer) & E32 (Hombre) \\
\hline
\end{tabular}

Fuente: Elaboración propia

Por último, cabe destacar que, para mejorar la comparativa, se han entrevistado a personas que tenían relación de parentesco entre sí. Dado que uno de los objetivos pre- 
tendidos es la comparación del capital social con el que cuentan adultos inmigrantes y jóvenes con pasado familiar migratorio reciente, hemos querido buscar a personas que compartieran la mayor parte de las variables que consideramos independientes para aislar el efecto de aquellas que realmente nos interesan. Contar con progenitores e hijos nos ha permitido realizar esta tarea con mayor calidad. Así:

\section{CUADRO 5}

Relación de parentesco entre entrevistados

\begin{tabular}{cc}
\hline Progenitor & Hijo/a \\
\hline E01 & E02 \\
E08 & E09 \\
E19 & E17 \\
E24 & E23 \\
E25 & E22 \\
\hline
\end{tabular}

Fuente: Elaboración propia

\section{Resultados obtenidos}

De forma habitual, las relaciones portadoras de capital social — aquellas en las que el acceso a apoyo material, información e influencia es habitual - tienen lugar con personas que se encuentran en una situación similar. Esto supone una menor incidencia en términos de movilidad social ascendente, dado que en aquellos casos en los que no hay un cierto excedente de recursos solo se puede ofrecer un apoyo de carácter ciertamente instrumental.

Únicamente aquellas personas que se encuentran en una situación de necesidad severa obtienen un beneficio relativamente importante de estas ayudas puntuales. Incluso cuando su valor objetivo no es elevado en términos absolutos, nos referimos a aquellos para los que poco puede resultar mucho.

El análisis del capital social (y de sus componentes) nos muestra ligeras diferencias entre grupos (Anexo - Tabla 3).

Los componentes del capital social con puntuaciones más elevadas son la influencia y la información (en menor medida). Por último, encontramos el apoyo material. Esta tendencia se da para todos los casos, aunque en los adultos se produce una mayor distancia, mientras que en los jóvenes (por tener apoyo material provisto por sus progenitores), se reducen las diferencias (Anexo - Tabla 4). 
En el caso concreto de los jóvenes, su posición socioeconómica viene determinada por la cantidad de recursos de capital social (apoyo económico, información e influencia) que tienen principalmente sus progenitores. Dado que el indicador que hemos utilizado es relativo (y capta la diferencia respecto a su posición inicial y no su valor absoluto), en su familia directa no encuentra un espacio de movilidad relevante (no se produce cambio de posición mientras dependan de ellos). Si sus relaciones en otros ámbitos son con personas en una posición similar, el aporte de recursos producido no se puede considerar sustancial.

Comparando los tres grupos, los jóvenes sin pasado migratorio familiar reciente poseen una puntuación intermedia en términos de capital social, produciéndose una suerte de equilibrio de fuerzas entre las relaciones con personas en una posición objetivamente más elevada (lo cual aumenta el capital social) y su punto de partida inicial (Anexo - Tabla 5).

Más allá de lo señalado, las comparativas entre jóvenes sin pasado migratorio o con él apenas arroja diferencias considerables, aunque desagregando de nuevo por casos vemos que las desviaciones típicas en los jóvenes sin pasado migratorio reciente son menores que en los que sí lo tienen; es decir, se da una mayor concentración en torno a la media en el caso de los jóvenes sin pasado migratorio que en los que sí lo tienen, donde la dispersión de valores es mucho mayor (Anexo - Tabla 6).

En términos generales, la mayoría de los casos analizados han obtenido puntuaciones más elevadas de bienes relacionales que de capital social. (Anexo - Tabla 7). Esto se debe principalmente a que las relaciones existentes son de calidad con la familia directa, los amigos y en ocasiones con la familia extensa, es decir, en aquellos grupos donde es más habitual acceder a afecto, valores e identidad (Anexo - Tabla 8).

El acceso a bienes relacionales se caracteriza por unos valores más elevados en afecto que en valores e identidad. Más allá de esta constatación, no existen patrones que permitan identificar variables (intensidad laboral, por ejemplo) que expliquen el comportamiento de estos indicadores. Únicamente la calidad y la frecuencia de las relaciones, especialmente en el seno de la familia cercana y de las amistades, son los parámetros que influyen directamente en este sentido.

El análisis agregado por grupos y subgrupos nos muestra que los adultos inmigrantes (Grupo 2) presentan valores más bajos que los otros dos. Obtienen puntuaciones más bajas en los tres componentes, pero, especialmente, en valores e identidad. Mientras que ellos proveen a sus hijos de ambos componentes, no los reciben en la misma medida. En todos los grupos el afecto es el componente con mayor puntuación relativa.

Los jóvenes sin pasado migratorio familiar reciente presentan valores algo más elevados que el resto de grupos y puntuaciones más homogéneas entre componentes, aunque también con valores más elevados para el afecto (Anexo - Tablas 9 y 10). 
Cuando llevamos a cabo la comparativa por grandes grupos, la tendencia se mantiene claramente $(\mathrm{BR}>\mathrm{CS}$ ) y se agudiza algo más la diferencia en el caso de los jóvenes españoles sin pasado migratorio. En parte, probablemente, eso es debido a la proximidad y el mayor arraigo de grupos proveedores de afecto, valores e identidad (familia, familia extensa, amigos...).

A continuación, procedemos a realizar un análisis de lo que aporta cada uno de los grupos de relación mencionados a la resultante de capital social (Anexo - Tabla 11). A la luz de los cálculos obtenidos y del análisis de las entrevistas en profundidad realizadas cabe destacar, en primer lugar, que es la familia directa (especialmente en los dos grupos de jóvenes) quien más contribuye al capital social. Esto se debe a que son los progenitores quienes sostienen en la mayoría de los casos la economía familiar, mientras que los jóvenes estudian o, en el mejor de los casos, contribuyen económicamente con un empleo cuya remuneración es relativamente débil dentro del conjunto de ingresos del hogar.

En el caso de los adultos, además de la familia directa (como ya hemos dicho, en menor medida que para los jóvenes), aparece la figura de las amistades como proveedores de alguno de los componentes que integran el capital social.

Destaca la aportación de la figura que hemos denominado «protector»o «valedor». A lo largo de las entrevistas nos hemos encontrado con que, a pesar de encontrarse en una situación económica precaria, y pese a no tener un entorno relacional afectivo excesivamente sólido, se daba la presencia de personas o instituciones cuya incidencia en términos de capital social (tanto de apoyo económico como de información) resultaba claramente significativa. Los aportes realizados por la figura del valedor suponen (comparativamente con el punto de partida de quien las recibe) unas contribuciones trascendentales.

Los aportes realizados por la familia directa presentan valores superiores para los jóvenes sin pasado migratorio, intermedios para los jóvenes con pasado familiar migratorio reciente, y claramente inferiores para los adultos.

Los amigos son la segunda fuente de recursos en términos de capital social, con claras diferencias entre nacionalidades: valores más elevados para ecuatorianos que para rumanos y, especialmente, para marroquíes (Anexo - Tablas 12 y 13).

En cuanto a los bienes relacionales, la familia directa es la proveedora mayoritaria de este tipo de bienes, el segundo lugar lo ocupa la familia extensa (en lugar de los amigos, como ocurría con el capital social tal y como se recoge en las Tablas 14 y 15 (Anexo).

Si bien los tres grupos presentan valores elevados en el grupo proveedor principal de bienes relacionales (familia directa), los dos grupos de jóvenes presentan valores similares en amigos y en familia extensa. En el caso de los adultos inmigrantes, las amistades son el segundo grupo por orden de relevancia. 
Es destacable la aportación del grupo «otros» en adultos inmigrantes a consecuencia - tal y como hemos mencionado- de las contribuciones realizadas por los valedores (Anexo - Tabla 16).

Por último, cabe destacar que el análisis por procedencia de origen (Anexo - Tabla 17) no aporta excesivos matices reseñables con las siguientes excepciones:

- Los casos relativos a Ecuador son los que cuentan con mayor puntuación global de capital social. Este procede (como en el resto de nacionalidades) principalmente de la familia directa y de los amigos, aunque son el único subgrupo que cuenta con un apoyo significativo por parte de la familia extensa y prácticamente nulo por parte de los vecinos.

- Por su parte, los subgrupos relativos a Marruecos son los que presentan puntuaciones más bajas de capital social. En este caso, el capital social proviene principalmente de la familia y en menor medida de los amigos y los vecinos.

- Los casos relativos a Rumanía presentan puntuaciones intermedias en relación a las otras dos nacionalidades. La procedencia de estos recursos se debe también mayoritariamente a la familia y en parte a los amigos, y muy poco por parte de la familia extensa.

En los tres casos, los componentes del capital social con puntuaciones más elevadas son la influencia y la información (en menor medida). Por último, encontramos el apoyo material.

ANEXO: TABLAS

TABLA 3

Capital social y sus componentes por grupos

\begin{tabular}{lcccc}
\hline & $\begin{array}{c}\text { Capital } \\
\text { social }\end{array}$ & $\begin{array}{c}\text { Apoyo } \\
\text { material }\end{array}$ & Información & Influencia \\
\hline $\begin{array}{l}\text { Jóvenes (con pasado migratorio familiar } \\
\text { reciente) }\end{array}$ & 9,36 & 2,87 & 3,29 & 3,21 \\
$\begin{array}{l}\text { Adultos (inmigrantes) } \\
\text { Jóvenes (sin pasado migratorio familiar } \\
\text { reciente) }\end{array}$ & 7,74 & 2,06 & 3,08 & 2,60 \\
\hline
\end{tabular}

Fuente: Elaboración propia 
TABLA 4

Componentes del capital social por subgrupos

\begin{tabular}{lccc}
\hline & Apoyo material & Información & Influencia \\
\hline G1 - Subgrupo 1.1 - Jóvenes (Ecuador) & 2,97 & 3,13 & 3,13 \\
G1 - Subgrupo 1.2 - Jóvenes (Marruecos) & 2,68 & 3,00 & 2,96 \\
G1 - Subgrupo 1.3 - Jóvenes (Rumanía) & 2,95 & 3,74 & 3,54 \\
G2 - Subgrupo 2.1 - Adultos (Ecuador) & 2,28 & 3,76 & 2,97 \\
G2 - Subgrupo 2.2 - Adultos (Marruecos) & 1,85 & 2,72 & 2,32 \\
G2 - Subgrupo 2.3 - Adultos (Rumanía) & 2,05 & 2,76 & 2,52 \\
G3 - Jóvenes (España) & 2,79 & 3,06 & 3,06 \\
\hline
\end{tabular}

Fuente: Elaboración propia

Tabla 5

Capital social y bienes relacionales por grupos

\begin{tabular}{lcc}
\hline & Capital social & $\begin{array}{c}\text { Bienes } \\
\text { relacionales }\end{array}$ \\
\hline G1. Jóvenes (pasado migratorio familiar reciente) & 9,36 & 10,13 \\
G2. Adultos (inmigrantes) & 7,74 & 7,87 \\
G3. Jóvenes (españoles) & 8,92 & 10,58 \\
\hline
\end{tabular}

Fuente: Elaboración propia

TABLA 6

Componentes del capital social por grupos

\begin{tabular}{llll}
\hline & Apoyo material & Información & Influencia \\
\hline $\begin{array}{llll}\text { G1. Jóvenes (pasado migratorio familiar } \\
\text { reciente) }\end{array}$ & 2,87 & 3,29 & 3,21 \\
G2. Adultos (inmigrantes) & 2,06 & 3,08 & 2,60 \\
G3. Jóvenes (españoles) & 2,79 & 3,06 & 3,06 \\
\hline
\end{tabular}

Fuente: Elaboración propia 
TABLA 7

Capital social y bienes relacionales por persona

\begin{tabular}{|c|c|c|c|}
\hline & Entrevista & Capital social & Bienes relacionales \\
\hline \multirow{4}{*}{$\begin{array}{c}\text { G1 } \\
\text { Subgrupo } 1.1 \\
\text { Jóvenes (Ecuador) }\end{array}$} & E07 (M) $\downarrow$ & 5,24 & 5,13 \\
\hline & $\mathrm{E} 22(\mathrm{~V}) \downarrow$ & 11,18 & 14,32 \\
\hline & $\mathrm{E} 27(\mathrm{M}) \uparrow$ & 9,68 & 10,83 \\
\hline & $\mathrm{E} 30(\mathrm{~V}) \uparrow$ & 10,76 & 10,83 \\
\hline \multirow{2}{*}{ G1 } & E04 (M) $\uparrow$ & 9,55 & 10,95 \\
\hline & $\mathrm{E} 17(\mathrm{~V}) \downarrow$ & 10,24 & 10,58 \\
\hline \multirow{3}{*}{$\begin{array}{c}\text { Subgrupo } 1.2 \\
\text { Jóvenes (Marruecos) }\end{array}$} & E20 (M) $\downarrow$ & 5,22 & 6,16 \\
\hline & $\mathrm{E} 33(\mathrm{~V}) \uparrow$ & 9,55 & 10,95 \\
\hline & E02 $(\mathrm{M}) \downarrow$ & 10,86 & 9,90 \\
\hline G1 & E09 (M) $\downarrow$ & 6,28 & 10,43 \\
\hline Subgrupo 1.3 & E16 (V) $\uparrow$ & 14,45 & 12,65 \\
\hline \multirow[t]{2}{*}{ Jóvenes (Rumanía) } & E23 (V) $\downarrow$ & 8,78 & 7,73 \\
\hline & E29 (M) $\uparrow$ & 10,81 & 11,53 \\
\hline \multirow{2}{*}{ G2 } & E11 $(\mathrm{M}) \uparrow$ & 10,40 & 10,28 \\
\hline & E12 $(\mathrm{M}) \uparrow$ & 9,37 & 9,14 \\
\hline \multirow{3}{*}{$\begin{array}{c}\text { Subgrupo } 2.1 \\
\text { Adultos (Ecuador) }\end{array}$} & E15 (V) $\downarrow$ & 8,90 & 8,30 \\
\hline & E25 (M) $\downarrow$ & 7,36 & 10,20 \\
\hline & E13 (M) $\downarrow$ & 3,88 & 2,74 \\
\hline G2 & $\mathrm{E} 14(\mathrm{~V}) \uparrow$ & 10,08 & 8,18 \\
\hline Subgrupo 2.2 & $\mathrm{E} 18(\mathrm{M}) \downarrow$ & 8,50 & 7,93 \\
\hline \multirow[t]{3}{*}{ Adultos (Marruecos) } & E19 (V) $\downarrow$ & 6,70 & 5,98 \\
\hline & $\mathrm{E} 21(\mathrm{~V}) \uparrow$ & 5,24 & 7,13 \\
\hline & $\mathrm{E} 01(\mathrm{M}) \downarrow$ & 6,56 & 6,74 \\
\hline G2 & $\mathrm{E} 05(\mathrm{~V}) \uparrow$ & 6,68 & 7,10 \\
\hline Subgrupo 2.3 & E08 (M) $\downarrow$ & 10,80 & 10,10 \\
\hline \multirow[t]{4}{*}{ Adultos (Rumanía) } & $\mathrm{E} 10(\mathrm{~V}) \uparrow$ & 4,85 & 7,20 \\
\hline & E24 (M) $\downarrow$ & 7,76 & 7,51 \\
\hline & E03 (V) $\uparrow$ & 11,30 & 14,85 \\
\hline & E06 $(\mathrm{M}) \uparrow$ & 8,20 & 9,15 \\
\hline G3 & E26 (V) $\downarrow$ & 6,80 & 7,05 \\
\hline \multirow[t]{3}{*}{ Jóvenes (España) } & E28 (M) $\downarrow$ & 8,96 & 8,49 \\
\hline & E31 (M) $\downarrow$ & 7,66 & 8,49 \\
\hline & E32 (V) $\uparrow$ & 10,58 & 15,43 \\
\hline
\end{tabular}

Fuente: Elaboración propia 
TABLA 8

Bienes relacionales y sus componentes por grupos

\begin{tabular}{lcccc}
\hline & $\begin{array}{c}\text { Bienes } \\
\text { relacionales }\end{array}$ & Afecto & Valores & Identidad \\
\hline $\begin{array}{l}\text { Jóvenes (con pasado migratorio familiar } \\
\text { reciente) }\end{array}$ & 10,13 & 3,56 & 3,17 & 3,40 \\
$\begin{array}{l}\text { Adultos (inmigrantes) } \\
\text { Jóvenes (sin pasado migratorio familiar } \\
\text { reciente) }\end{array}$ & 7,87 & 3,37 & 2,42 & 2,08 \\
\hline
\end{tabular}

Fuente: Elaboración propia

TABLA 9

Bienes relacionales por subgrupo

\begin{tabular}{llll}
\hline & Afecto & Valores & Identidad \\
\hline G1 - Subgrupo 1.1 - Jóvenes (Ecuador) & 3,80 & 2,99 & 3,49 \\
G1 - Subgrupo 1.2 - Jóvenes (Marruecos) & 3,31 & 3,02 & 3,33 \\
G1 - Subgrupo 1.3 - Jóvenes (Rumanía) & 3,57 & 3,50 & 3,37 \\
G2 - Subgrupo 2.1 - Adultos (Ecuador) & 4,06 & 3,00 & 2,42 \\
G2 - Subgrupo 2.2 - Adultos (Marruecos) & 2,68 & 1,97 & 1,75 \\
G2 - Subgrupo 2.3 - Adultos (Rumanía) & 3,37 & 2,30 & 2,06 \\
G3 - Jóvenes (España) & 3,82 & 3,19 & 3,57 \\
\hline
\end{tabular}

Fuente: Elaboración propia

TABLA 10

Bienes relacionales por grupo

\begin{tabular}{lccc}
\hline & Afecto & Valores & Identidad \\
\hline G1. Jóvenes (pasado migratorio familiar reciente) & 3,56 & 3,17 & 3,40 \\
G2. Adultos (inmigrantes) & 3,37 & 2,42 & 2,08 \\
G3. Jóvenes (españoles) & 3,82 & 3,19 & 3,57 \\
\hline
\end{tabular}

Fuente: Elaboración propia 
TABLA 11

Aporte al capital social de cada ámbito de relación por persona

\begin{tabular}{|c|c|c|c|c|c|c|}
\hline & Entrevista & $\begin{array}{c}\text { Familia } \\
\text { directa }\end{array}$ & $\begin{array}{l}\text { Familia } \\
\text { extensa }\end{array}$ & Amigos/as & Vecinos/as & Otros \\
\hline \multirow{4}{*}{$\begin{array}{c}\text { G1 } \\
\text { Subgrupo 1.1 } \\
\text { Jóvenes (Ecuador) }\end{array}$} & E07 (M) $\downarrow$ & 3,00 & 1,80 & 0,26 & 0,18 & 0,00 \\
\hline & $\mathrm{E} 22(\mathrm{~V}) \downarrow$ & 6,00 & 2,40 & 2,60 & 0,18 & 0,00 \\
\hline & $\mathrm{E} 27(\mathrm{M}) \uparrow$ & 6,00 & 0,90 & 2,60 & 0,18 & 0,00 \\
\hline & $\mathrm{E} 30(\mathrm{~V}) \uparrow$ & 6,00 & 1,80 & 2,60 & 0,36 & 0,00 \\
\hline \multirow{5}{*}{$\begin{array}{c}\text { G1 } \\
\text { Subgrupo } 1.2 \\
\text { Jóvenes (Marruecos) }\end{array}$} & E04 (M) $\uparrow$ & 6,00 & 0,45 & 1,30 & 1,80 & 0,00 \\
\hline & $\mathrm{E} 17(\mathrm{~V}) \downarrow$ & 6,00 & 0,36 & 2,60 & 0,90 & 0,38 \\
\hline & $\mathrm{E} 20(\mathrm{M}) \downarrow$ & 3,00 & 0,36 & 1,30 & 0,18 & 0,38 \\
\hline & E33 $(V) \uparrow$ & 6,00 & 0,45 & 1,30 & 1,80 & 0,00 \\
\hline & $\mathrm{E} 02(\mathrm{M}) \downarrow$ & 6,00 & 0,18 & 2,60 & 0,18 & 1,90 \\
\hline G1 & E09 (M) $\downarrow$ & 3,00 & 1,80 & 1,30 & 0,18 & 0,00 \\
\hline Subgrupo 1.3 & E16 $(\mathrm{V}) \uparrow$ & 6,00 & 0,45 & 5,20 & 0,90 & 1,90 \\
\hline \multirow[t]{2}{*}{ Jóvenes (Rumanía) } & $\mathrm{E} 23(\mathrm{~V}) \downarrow$ & 6,00 & 0,00 & 2,60 & 0,18 & 0,00 \\
\hline & E29 (M) $\uparrow$ & 6,00 & 0,90 & 2,60 & 0,36 & 0,95 \\
\hline \multirow{5}{*}{$\begin{array}{c}\text { G2 } \\
\text { Subgrupo 2.1 } \\
\text { Adultos (Ecuador) }\end{array}$} & E11 (M) $\uparrow$ & 1,50 & 0,90 & 5,20 & 0,90 & 1,90 \\
\hline & $\mathrm{E} 12(\mathrm{M}) \uparrow$ & 3,00 & 0,09 & 2,60 & 0,18 & 3,50 \\
\hline & $\mathrm{E} 15(\mathrm{~V}) \downarrow$ & 3,00 & 0,00 & 1,80 & 0,90 & 3,20 \\
\hline & E25 (M) $\downarrow$ & 3,00 & 1,20 & 2,60 & 0,18 & 0,38 \\
\hline & $\mathrm{E} 13(\mathrm{M}) \downarrow$ & 0,00 & 1,80 & 0,00 & 0,18 & 1,90 \\
\hline G2 & E14 (V) $\uparrow$ & 0,00 & 0,90 & 5,20 & 0,18 & 3,80 \\
\hline Subgrupo 2.2 & $\mathrm{E} 18(\mathrm{M}) \downarrow$ & 3,00 & 1,80 & 0,00 & 1,80 & 1,90 \\
\hline \multirow[t]{3}{*}{ Adultos (Marruecos) } & E19 (V) $\downarrow$ & 3,00 & 0,00 & 0,00 & 1,80 & 1,90 \\
\hline & E21 (V) $\uparrow$ & 3,00 & 1,80 & 0,26 & 0,18 & 0,00 \\
\hline & E01 (M) $\downarrow$ & 3,00 & 0,18 & 1,30 & 0,18 & 1,90 \\
\hline G2 & E05 $(\mathrm{V}) \uparrow$ & 3,00 & 0,18 & 2,60 & 0,90 & 0,00 \\
\hline Subgrupo 2.3 & E08 (M) $\downarrow$ & 3,00 & 0,90 & 1,30 & 1,80 & 3,80 \\
\hline \multirow[t]{4}{*}{ Adultos (Rumanía) } & E10 $(\mathrm{V}) \uparrow$ & 3,00 & 0,18 & 1,30 & 0,18 & 0,19 \\
\hline & E24 (M) $\downarrow$ & 3,00 & 0,36 & 2,60 & 1,80 & 0,00 \\
\hline & E03 $(\mathrm{V}) \uparrow$ & 6,00 & 1,80 & 2,60 & 0,90 & 0,00 \\
\hline & E06 (M) $\uparrow$ & 6,00 & 0,00 & 1,30 & 0,90 & 0,00 \\
\hline G3 & E26 $(\mathrm{V}) \downarrow$ & 6,00 & 0,36 & 0,26 & 0,18 & 0,00 \\
\hline \multirow[t]{3}{*}{ Jóvenes (España) } & E28 (M) $\downarrow$ & 6,00 & 0,18 & 2,60 & 0,18 & 0,00 \\
\hline & $\mathrm{E} 31(\mathrm{M}) \downarrow$ & 6,00 & 0,18 & 1,30 & 0,18 & 0,00 \\
\hline & E32 $(\mathrm{V}) \uparrow$ & 6,00 & 1,80 & 0,00 & 0,18 & 0,00 \\
\hline
\end{tabular}

Fuente: Elaboración propia 


\section{TABLA 12}

Aporte al capital social de cada ámbito de relación por subgrupo

\begin{tabular}{lccccc}
\hline & $\begin{array}{c}\text { Familia } \\
\text { directa }\end{array}$ & $\begin{array}{c}\text { Familia } \\
\text { extensa }\end{array}$ & $\begin{array}{c}\text { Amigos/ } \\
\text { as }\end{array}$ & $\begin{array}{c}\text { Vecinos/ } \\
\text { as }\end{array}$ & Otros \\
\hline G1 - Subgrupo 1.1 - Jóvenes (Ecuador) & 5,25 & 1,73 & 2,02 & 0,23 & 0,00 \\
G1 - Subgrupo 1.2 - Jóvenes (Marruecos) & 5,25 & 0,41 & 1,63 & 1,17 & 0,19 \\
G1 - Subgrupo 1.3 - Jóvenes (Rumanía) & 5,40 & 0,67 & 2,86 & 0,36 & 0,95 \\
G2 - Subgrupo 2.1 - Adultos (Ecuador) & 2,63 & 0,55 & 3,05 & 0,54 & 2,25 \\
G2 - Subgrupo 2.2 - Adultos (Marruecos) & 1,80 & 1,26 & 1,09 & 0,83 & 1,90 \\
G2 - Subgrupo 2.3 - Adultos (Rumanía) & 3,00 & 0,36 & 1,82 & 0,97 & 1,18 \\
G3 - Jóvenes (España) & 6,00 & 0,72 & 1,34 & 0,42 & 0,00 \\
\hline
\end{tabular}

Fuente: Elaboración propia

\section{TABLA 13}

Aporte al capital social de cada ámbito de relación por grupo

\begin{tabular}{lllllc}
\hline & $\begin{array}{c}\text { Familia } \\
\text { directa }\end{array}$ & $\begin{array}{l}\text { Familia } \\
\text { extensa }\end{array}$ & $\begin{array}{c}\text { Amigos/ } \\
\text { as }\end{array}$ & $\begin{array}{c}\text { Vecinos/ } \\
\text { as }\end{array}$ & Otros \\
\hline G1. Jóvenes (pasado migratorio familiar & 5,30 & 0,93 & 2,17 & 0,59 & 0,38 \\
reciente) & & & & & \\
G2. Adultos (inmigrantes) & 2,48 & 0,72 & 1,99 & 0,78 & 1,77 \\
G3. Jóvenes (españoles) & 6,00 & 0,72 & 1,34 & 0,42 & 0,00 \\
\hline
\end{tabular}

Fuente: Elaboración propia 
TABLA 14

Aporte a los bienes relacionales de cada ámbito de relación por persona

\begin{tabular}{|c|c|c|c|c|c|c|}
\hline & Entrevista & $\begin{array}{c}\text { Familia } \\
\text { directa }\end{array}$ & $\begin{array}{l}\text { Familia } \\
\text { extensa }\end{array}$ & Amigos/as & Vecinos/as & Otros \\
\hline \multirow{4}{*}{$\begin{array}{c}\text { G1 } \\
\text { Subgrupo 1.1 } \\
\text { Jóvenes (Ecuador) }\end{array}$} & - & 2,00 & 2,60 & 0,30 & 0,23 & 0,00 \\
\hline & E22 (V) $\downarrow$ & 6,00 & 5,20 & 3,00 & 0,12 & 0,00 \\
\hline & $\mathrm{E} 27(\mathrm{M}) \uparrow$ & 6,00 & 2,60 & 2,00 & 0,23 & 0,00 \\
\hline & $\mathrm{E} 30(\mathrm{~V}) \uparrow$ & 6,00 & 2,60 & 2,00 & 0,23 & 0,00 \\
\hline \multirow{5}{*}{$\begin{array}{l}\text { G1 } \\
\text { grupo } 1.2 \\
\text { (Marruecos) }\end{array}$} & E04 (M) $\uparrow$ & 6,00 & 1,80 & 2,00 & 1,15 & 0,00 \\
\hline & E17 (V) $\downarrow$ & 6,00 & 0,26 & 3,00 & 1,15 & 0,17 \\
\hline & $\mathrm{E} 20(\mathrm{M}) \downarrow$ & 4,00 & 0,26 & 1,50 & 0,23 & 0,17 \\
\hline & $\mathrm{E} 33(\mathrm{~V}) \uparrow$ & 6,00 & 1,80 & 2,00 & 1,15 & 0,00 \\
\hline & E02 $(\mathrm{M}) \downarrow$ & 6,00 & 0,52 & 1,50 & 0,23 & 1,65 \\
\hline G1 & E09 (M) $\downarrow$ & 3,50 & 5,20 & 1,50 & 0,23 & 0,00 \\
\hline \multirow{3}{*}{ Jóvenes (Rumanía) } & $\mathrm{E} 16(\mathrm{~V}) \uparrow$ & 6,00 & 1,30 & 3,00 & 1,15 & 1,20 \\
\hline & E23 (V) $\downarrow$ & 6,00 & 0,00 & 1,50 & 0,23 & 0,00 \\
\hline & $\mathrm{E} 29(\mathrm{M}) \uparrow$ & 6,00 & 2,60 & 1,50 & 0,23 & 1,20 \\
\hline \multirow{5}{*}{$\begin{array}{c}\text { G2 } \\
\text { Subgrupo 2.1 } \\
\text { Adultos (Ecuador) }\end{array}$} & $\mathrm{E} 11(\mathrm{M}) \uparrow$ & 3,00 & 1,30 & 4,00 & 1,15 & 0,83 \\
\hline & $\mathrm{E} 12(\mathrm{M}) \uparrow$ & 4,00 & 0,26 & 3,00 & 0,23 & 1,65 \\
\hline & $\mathrm{E} 15(\mathrm{~V}) \downarrow$ & 4,00 & 0,00 & 1,50 & 1,15 & 1,65 \\
\hline & E25 (M) $\downarrow$ & 4,00 & 1,80 & 4,00 & 0,23 & 0,17 \\
\hline & $\mathrm{E} 13(\mathrm{M}) \downarrow$ & 0,00 & 1,80 & 0,00 & 0,12 & 0,83 \\
\hline G2 & $\mathrm{E} 14(\mathrm{~V}) \uparrow$ & 0,00 & 2,30 & 4,00 & 0,23 & 1,65 \\
\hline Subgrupo 2.2 & $\mathrm{E} 18(\mathrm{M}) \downarrow$ & 4,00 & 1,80 & 0,00 & 1,30 & 0,83 \\
\hline \multirow[t]{3}{*}{ Adultos (Marruecos) } & E19 (V) $\downarrow$ & 4,00 & 0,00 & 0,00 & 1,15 & 0,83 \\
\hline & $\mathrm{E} 21(\mathrm{~V}) \uparrow$ & 4,00 & 2,60 & 0,30 & 0,23 & 0,00 \\
\hline & $\mathrm{E} 01(\mathrm{M}) \downarrow$ & 3,00 & 0,36 & 1,50 & 0,23 & 1,65 \\
\hline G2 & E05 (V) $\uparrow$ & 4,00 & 0,52 & 2,00 & 0,58 & 0,00 \\
\hline Subgrupo 2.3 & $\mathrm{E} 08(\mathrm{M}) \downarrow$ & 4,00 & 1,80 & 1,50 & 1,15 & 1,65 \\
\hline \multirow[t]{4}{*}{ Adultos (Rumanía) } & $\mathrm{E} 10(\mathrm{~V}) \uparrow$ & 4,00 & 0,52 & 2,50 & 0,12 & 0,06 \\
\hline & E24 (M) $\downarrow$ & 4,00 & 0,36 & 2,00 & 1,15 & 0,00 \\
\hline & E03 (V) $\uparrow$ & 6,00 & 5,20 & 2,50 & 1,15 & 0,00 \\
\hline & E06 (M) $\uparrow$ & 6,00 & 0,00 & 2,00 & 1,15 & 0,00 \\
\hline G3 & E26 (V) $\downarrow$ & 6,00 & 0,52 & 0,30 & 0,23 & 0,00 \\
\hline \multirow[t]{3}{*}{ Jóvenes (España) } & E28 (M) $\downarrow$ & 6,00 & 0,26 & 2,00 & 0,23 & 0,00 \\
\hline & E31 (M) $\downarrow$ & 6,00 & 0,26 & 2,00 & 0,23 & 0,00 \\
\hline & $\mathrm{E} 32(\mathrm{~V}) \uparrow$ & 6,00 & 5,20 & 4,00 & 0,23 & 0,00 \\
\hline
\end{tabular}

Fuente: Elaboración propia 
TABLA 15

Aporte a los bienes relacionales cada ámbito de relación por subgrupo

\begin{tabular}{llllll}
\hline & $\begin{array}{c}\text { Familia } \\
\text { directa }\end{array}$ & $\begin{array}{c}\text { Familia } \\
\text { extensa }\end{array}$ & $\begin{array}{c}\text { Amigos/ } \\
\text { as }\end{array}$ & $\begin{array}{c}\text { Vecinos/ } \\
\text { as }\end{array}$ & Otros \\
\hline G1 - Subgrupo 1.1 - Jóvenes (Ecuador) & 5,00 & 3,25 & 1,83 & 0,20 & 0,00 \\
G1 - Subgrupo 1.2 - Jóvenes (Marruecos) & 5,50 & 1,03 & 2,13 & 0,92 & 0,08 \\
G1 - Subgrupo 1.3 - Jóvenes (Rumanía) & 5,50 & 1,92 & 1,80 & 0,41 & 0,81 \\
G2 - Subgrupo 2.1 - Adultos (Ecuador) & 3,75 & 0,84 & 3,13 & 0,69 & 1,07 \\
G2 - Subgrupo 2.2 - Adultos (Marruecos) & 2,40 & 1,70 & 0,86 & 0,61 & 0,83 \\
G2 - Subgrupo 2.3 - Adultos (Rumanía) & 3,80 & 0,71 & 1,90 & 0,64 & 0,67 \\
G3 - Jóvenes (España) & 6,00 & 1,91 & 2,13 & 0,54 & 0,00
\end{tabular}

TABLA 16

Aporte a los bienes relacionales de cada ámbito de relación por grupo

\begin{tabular}{lccccc}
\hline & $\begin{array}{c}\text { Familia } \\
\text { directa }\end{array}$ & $\begin{array}{c}\text { Familia } \\
\text { extensa }\end{array}$ & $\begin{array}{c}\text { Amigos/ } \\
\text { as }\end{array}$ & $\begin{array}{c}\text { Vecinos/ } \\
\text { as }\end{array}$ & Otros \\
\hline $\begin{array}{l}\text { G1. Jóvenes (pasado migratorio familiar } \\
\text { reciente) }\end{array}$ & 5,33 & 2,07 & 1,92 & 0,51 & 0,30 \\
G2. Adultos (inmigrantes) & 3,32 & 1,08 & 1,96 & 0,65 & 0,86 \\
G3. Jóvenes (españoles) & 6,00 & 1,91 & 2,13 & 0,54 & 0,00 \\
\hline
\end{tabular}

Fuente: Elaboración propia

TABLA 17

Capital social y bienes relacionales por subgrupos

\begin{tabular}{lcc}
\hline & Capital social & Bienes relacionales \\
\hline G1 - Subgrupo 1.1 - Jóvenes (Ecuador) & 9,22 & 10,28 \\
G1 - Subgrupo 1.2 - Jóvenes (Marruecos) & 8,64 & 9,66 \\
G1 - Subgrupo 1.3 - Jóvenes (Rumanía) & 10,24 & 10,45 \\
G2 - Subgrupo 2.1 - Adultos (Ecuador) & 9,01 & 9,48 \\
G2 - Subgrupo 2.2 - Adultos (Marruecos) & 6,88 & 6,39 \\
G2 - Subgrupo 2.3 - Adultos (Rumanía) & 7,33 & 7,73 \\
G3 - Jóvenes (España) & 8,92 & 10,58 \\
\hline
\end{tabular}

Fuente: Elaboración propia 


\section{BIBLIOGRAFÍA}

BOLÍVAR, G. A. y ELIZALDE, A. (2011): «Capital social y capital», en Polis, Revista de la Universidad Bolivariana, n. ${ }^{\circ}$ 29, 2011, pp. 7-16.

BOURDIEU, P. (1980): «Le capital social, notes provisoires», Actes de la Recherche en Sciences Sociales, enero, n. ${ }^{\circ} 31$.

COLEMAN, J. (1990): Foundations of Social Theory. Cambridge: Harvard University Press.

FUKUYAMA, F. (1995b): «Social Capital and the Global Economy: A Redrawn Map of the World», en Foreign Affairs, n. ${ }^{\circ} 74$.

GARCÍA FERRNADO, M. (2008): «El capital social como variable sociológica y el espíritu del capitalismo», en MEIL LANDWERLIN, G. y TORRES ALBERO, C. (coords.), Sociología y realidad social. Libro homenaje a Miguel Beltrán Villalva. Madrid: Centro de investigaciones Sociológicas.

GRANOVETTER, M. S. (1973): «The strength of weak ties», American Journal of Sociology, n. ${ }^{\circ} 6$.

LIN, N. (1999): «Building a Theory of Social Capital» en Connections, n. 22.

LORENZO, F. (2015): Vínculos sociales e integración: estudio sobre el capital social de los hijos de inmigrantes procedentes de Rumanía, Ecuador y Marruecos en España. Tesis Doctoral. Madrid: UNED. Departamento de Sociología III (Tendencias Sociales).

LORENZO, F. (2017): «¿Capital social o bienes relacionales? Elaboración de una propuesta conceptual del capital social» en Documentación Social n. ${ }^{\circ}$ XX. Cáritas Editores. Madrid.

OSTROM, E. Y AHN, T. K. (2003): «Una perspectiva del capital social desde las ciencias sociales: capital social y acción colectiva», en Revista Mexicana de Sociología, vol. 65, n. $^{\circ} 1$.

PORTES, A. (1998): «Social capital: its origins and applications in modern sociology», Annual Review of Sociology, n. ${ }^{\circ} 24$.

PUTNAM, R. D. et al. (1993): Making Democracy Work: Civic Traditions in Modern Italy. Princeton, NJ: Princeton University Press.

PUTNAM, R. D. (2002): Solo en la bolera. Colapso y resurgimiento de la comunidad norteamericana. Barcelona: Galaxia Gutemberg.

PUTNAM, R. D. (2003): El declive del capital social. Un estudio internacional sobre sociedades y el sentido comunitario. Barcelona: Galaxia Gutemberg.

WELLMAN, B. (1991): "Which types of ties and networks provide what kind of social support?» en LAWLER, E .J. et al., Advances in Group Processes, n. ${ }^{\circ}$ 9. Bingley, UK: Emerald. 